\title{
Orthogonal 3-D Graph Drawing *
}

\author{
T. Biedl ${ }^{1}$ and T. Shermer ${ }^{2}$ and S. Whitesides ${ }^{1}$ and S. Wismath ${ }^{3}$ \\ 1. School of Computer Science, McGill University, Montreal, PQ H3A2A7, Canada \\ 2 School of Computing Science, Simon Fraser University, Burnaby, BC V5A1A6, \\ Canada \\ 3 Department of Mathematics and Computer Science, University of Lethbridge, \\ Lethbridge, AB T1K3M4, Canada
}

\begin{abstract}
This paper studies 3-D orthogonal grid drawings for graphs of arbitrary degree, $K_{n}$ in particular, with vertices drawn as boxes. It establishes an asymptotic lower bound for the volume of the bounding box of such drawings and exhibits a construction that achieves this bound. No edge route in this unconstrained construction bends more than three times.

For drawings constrained to have at most $k$ bends on any edge route, simple constructions are given for $k=1$ and $k=2$. The unconstrained construction handles the $k \geq 3$ cases, while for $k=0$ (no bends), it is proved here that not all graphs can be drawn.
\end{abstract}

\section{Introduction}

This paper offers methods for constructing 3-D orthogonal grid drawings for graphs of arbitrary degree. It also contributes a lower bound result for the volumes of such drawings, establishing that one of our constructions is in some sense optimal. To state the main results clearly, we explain, following some terminology, the drawing conventions and volume measure used.

A grid point is a point in $R^{3}$ whose coordinates are all integers. A grid box is the set of all points $(x, y, z)$ in $R^{3}$ satisfying $x_{0} \leq x \leq x_{1}, y_{0} \leq y \leq y_{1}$ and $z_{0} \leq z \leq z_{1}$ for some integers $x_{0}, x_{1}, y_{0}, y_{1}, z_{0}, z_{1}$. A port of a box is any grid point of the box that is extremal in at least one direction. A grid box is said to have dimensions $a \times b \times c$ whenever $x_{1}=x_{0}+a-1, y_{1}=y_{0}+b-1$, and $z_{1}=z_{0}+c-1$. The volume of such a box is defined to be the number of grid points it contains, namely $a b c$. For example, a single grid point is a $1 \times 1 \times 1$ box of volume 1 . The volume of a drawing is the volume of its bounding box, which is the smallest volume grid box containing the drawing. Often we refer to the bounding box as an $X \times Y \times Z$-grid.

Throughout this paper, a $3-D$ orthogonal grid drawing of a graph $G=(V, E)$ is a drawing that satisfies the following. Distinct vertices of $V$ are represented

\footnotetext{
* The authors gratefully thank N.S.E.R.C. for financial assistance.
} 
by disjoint grid boxes ${ }^{4}$. An edge $e=\left(v_{1}, v_{2}\right)$ of $E$ is drawn as a simple path that follows grid lines, possibly turning ("bending") at grid points; the endpoints of the path for $e$ are ports on the boxes representing $v_{1}$ and $v_{2}$. The intermediate points along the path for an edge do not belong to any vertex box, nor do they belong to any other edge path. See Figure 1. In what follows, graph theoretic terms such as vertex are typically used to refer both to the graph theoretic object and to its representation in a drawing.

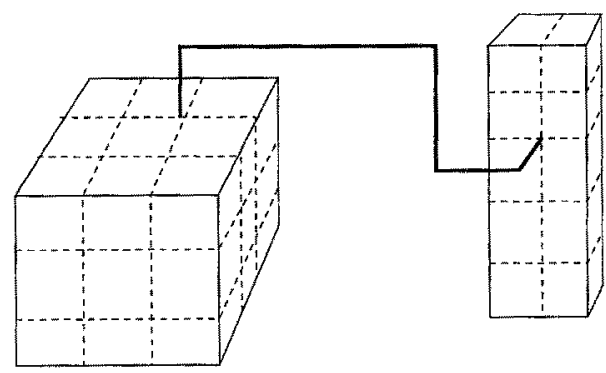

Fig. 1. Two boxes joined by a 4-bend edge.

For graphs drawn orthogonally in the 2-D grid, early research mainly considered graphs of maximum degree 4 and represented vertices as single grid points. More recently, 2-D orthogonal grid drawings of higher degree graphs have been investigated, where vertices have been drawn as rectangular boxes. See for example [FK96], [PT96], [BMT97].

At present, there are few results on 3-D orthogonal grid drawings. Rosenberg showed that any graph of maximum degree 6 can be embedded in a 3-D grid of volume $\mathcal{O}\left(n^{3 / 2}\right)$, and that this is asymptotically optimal [Ros83]. No bounds on the number of bends were given. Recently, Eades, Symvonis and Whitesides gave a method for drawing graphs of maximum degree 6 in a grid of side-length $4 \sqrt{n}$, with vertices represented by single grid points and each edge having at most 7 bends [ESW97]. They also gave a simple method for drawing such graphs in a grid of side-length $3 n$, creating at most 3 bends on each edge. Papakostas and Tollis have proposed a more elaborate method that produces a drawing of volume at most $4.66 n^{3}$ [PT97].

The focus of this paper is on 3-D orthogonal grid drawings of complete graphs. Since any simple graph $G$ on $n$ vertices is a subgraph of the complete graph $K_{n}$, a drawing of $K_{n}$ immediately provides a drawing for $G$, since irrelevant edges may be deleted from the drawing. Complete graphs are also critical for many lower bound arguments.

\footnotetext{
${ }^{4}$ This paper allows vertices to be represented by degenerate boxes, i.e., by boxes that have dimension 1 with respect to one or more coordinate directions. Such degeneracies can be removed by adding additional grid lines, which increases the volume of the drawing by a multiplicative constant.
} 
convention: From now on, the terms drawing and 3-D orthogonal grid drawing are used interchangably.

In informal language, the main results of this paper are as follows.

- For all sufficiently large $n, K_{n}$ has no bend-free drawing.

- Any drawing of $K_{n}$ has volume $\Omega\left(n^{2.5}\right)$.

- $K_{n}$ can be drawn in $O\left(n^{3}\right)$ volume with at most $k=1$ bend per edge.

- $K_{n}$ can be drawn in $O\left(n^{3}\right)$ volume with at most $k=2$ bends per edge.

- $K_{n}$ can be drawn in $O\left(n^{2.5}\right)$ volume with at most $k=3$ bends per edge.

Note that for $k \geq 3$, the upper and lower bounds on the volume match (within a constant factor) when a maximum of $k$ bends per edge is allowed. The constructions of this paper have reasonably small constant factors for the volume. Only for the $k=1$ and $k=2$ cases do the bounds not match; in each of these cases we give an $O\left(n^{3}\right)$ volume drawing of $K_{n}$ and leave as an open problem whether this drawing indeed has asymptotically optimum volume.

The results can be restated more precisely with the following terminology.

definition: Let $\operatorname{vol}(n)$ denote the minimum possible volume of any drawing of $K_{n}$, and let $\operatorname{vol}_{k}(n)$ denote the minimum possible volume for drawings of $K_{n}$ that have $k$ or fewer bends on any edge.

In these terms, the main results are that $v o l_{0}(n)$ is undefined for large $n$, $\operatorname{vol}(n)$ is in $\Omega\left(n^{2.5}\right), \operatorname{vol}_{1}(n)$ and $\operatorname{vol}_{2}(n)$ are in $O\left(n^{3}\right)$, and $\operatorname{vol}_{k}(n)$ is in $\Theta\left(n^{2.5}\right)$ for $k \geq 3$.

\section{No Bends}

The main result of this section is that there exist graphs that have no 0-bend 3-D orthogonal drawing. If no bends are permitted in the drawing, then the edges correspond to axis-parallel visibility lines between pairs of boxes. Such visibility representations have been studied in 2-D by Wismath [Wis85] and by Tamassia and Tollis [TT86], and in 3-D with 2-D objects [BEFLMRSW93], [FHW96]. A 3D orthogonal drawing of a graph with no bends splits the edges into three classes, depending on the direction of visibility. Each class of edges forms a graph that has a visibility representation using only one direction of visibility. Our lower bound result depends on the fact that $K_{56}$ has no such visibility representation, as shown by [FHW96].

The 3-Ramsey number $R(r, b, g)$ is the smallest number such that any arbitrary colouring of the edges of $K_{R(r, b, g)}$ with colours red, blue and green induces either a red $K_{r}$, or a blue $K_{b}$, or a green $K_{g}$ as a subgraph. This number exists and is finite; see for example [GRS80].

Theorem 1. For all sufficiently large $n$ (e.g., $n \geq R(56,56,56)$ ), $K_{n}$ has no bend-free 3-D orthogonal grid drawing.

One consequence of the previous theorem is that $\Omega\left(n^{2}\right)$ bends are required in any 3-D orthogonal grid drawing of $K_{n}$. Details are omitted. 


\section{A Lower Bound on the Volume}

Recall that $\operatorname{vol}(n)$ is the minimum possible volume for a drawing of $K_{n}$. This definition is valid since, as later sections show, every $K_{n}$ has a drawing if edges are allowed to bend.

A $z$-line is a line that is parallel to the $z$-axis; $y$-lines and $x$-lines are defined analogously. A $z$-plane is a plane that is orthogonal to the $z$-axis; $x$-planes and $y$-planes are defined analogously.

Theorem 2. $\operatorname{vol}(n) \in \Omega\left(n^{2.5}\right)$.

Proof. The constants that appear below were chosen for convenience and have no special significance other than that they give a simple proof. We make no attempt here to produce a large constant multiplier for the $n^{2.5}$.

Consider a drawing of $K_{n}$ in a grid of dimensions $X \times Y \times Z$.

\section{Case 1: A line intersects many vertices}

Assume there exists a $z$-line intersecting at least $t$ vertices, where $t$ is even and $t \geq \frac{1}{16} n$. Let $v_{1}, \ldots, v_{t}$ be any $t$ of the vertices intersected by the $z$-line, listed in order of occurrence along the line, and let $P_{z}$ be a $z$-plane (not necessarily with integer $z$-coordinate) that intersects none of these $t$ vertices and that separates the first half of them from the second half.

Since the $\frac{1}{4} t^{2}$ edges connecting these two groups must cross the plane $P_{z}$, this plane must contain at least $\frac{1}{4} t^{2}$ points having integer $x$ - and $y$-coordinates. Hence $X Y \geq \frac{1}{4} t^{2}>\frac{1}{1024} n^{2}$. Also, $Z \geq \frac{1}{16} n$ since the $z$-line intersects at least $\frac{1}{16} n$ vertices. Thus vol $(n)>\frac{1}{16384} n^{3}$.

\section{Case 2: A plane intersects many vertices}

Assume now that no $x$-line, $y$-line or $z$-line intersects as many as $\frac{1}{16} n$ vertices, but that there exists a $z$-plane $P_{z}$ intersecting at least $\frac{1}{4} n$ vertices.

A vertex is left of an $x$-plane $P_{x}$ if all the points in its grid box have $x$ ccordinates less than $x$. The notion of right of $P_{x}$ is analogous. As $P_{x}$ is swept from smaller to larger values of $x$, the $y$-line determined by its intersection with $P_{z}$ intersects fewer than $\frac{1}{16} n$ vertices, by assumption. As $x$ increases, an integer $x=x_{0}$ is encountered where, for the last time, there are fewer than $\frac{1}{16} n$ vertices left of $P_{x}$ and intersecting $P_{z}$.

The number of vertices that intersect $P_{z}$ and that lie left of $P_{x_{0}+1}$ is at least $\frac{1}{16} n$ but at most $\frac{2}{16} n-2$. Thus at least $\frac{1}{16} n$ vertices intersect $P_{z}$ and lie right of $P_{x_{0}+1}$. There are at least $\frac{1}{256} n^{2}$ edges between the vertices on the left and the vertices on the right, so $Y Z \geq \frac{1}{256} n^{2}$. Apply exactly the same argument in the $y$-direction to obtain $X Z \geq \frac{1}{256} n^{2}$. Finally, note that $X Y \geq \frac{1}{4} n$, since $P_{z}$ intersects $\frac{1}{4} n$ vertices. Consequently, $X Y Z=\sqrt{Y Z \cdot X Z \cdot X Y} \geq \frac{1}{312} n^{5 / 2}$.

\section{Case 3: No plane intersects many vertices}

Assume now that no plane intersects as many as $\frac{1}{4} n$ vertices. Consider $P_{x}$ planes in order of increasing $x$ value. By an argument analogous to the one in Case 2, a $P_{x}$ will be encountered for which at least $\frac{1}{4} n$ vertices lie left of $P_{x}$, and at least $\frac{1}{4} n$ vertices lie right of $P_{x}$. Consequently, $P_{x}$ contains at least $\frac{1}{16} n^{2}$ points 
with integer $y$ - and $z$-coordinates, and $Y Z \geq \frac{1}{16} n^{2}$. Since the same argument holds for the other two directions, $X Y Z \geq\left(\frac{1}{16} n^{2}\right)^{3 / 2}=\frac{1}{64} n^{3}$.

For all sufficiently large $n$, the bound given by Case 2 is the smallest of the three; hence $\operatorname{vol}(n) \in \Omega\left(n^{5 / 2}\right)$.

\section{Constructions}

The lower bound of the previous section provides a volumetric goal for layout strategies. This section presents a construction that achieves this lower bound with a small constant factor. For the $k=1$ case, two strategies are described and then modified to give a drawing for the $k=2$ case. A simple construction that realizes the $\Omega\left(n^{2.5}\right)$ lower bound for volume is described in subsection 4.3. The construction generates at most 3 bends on any edge and hence is valid for each $k \geq 3$. Whether the lower bound is attainable when $k=1$ or 2 remains an open problem.

In each of the constructions, vertices are first placed as points in a 2-D $x, y$-plane. Next, all the edges are routed in the same $x y$-plane, with overlap and crossings of edges temporarily permitted. Then a number $Z$ of $z$-planes is introduced, and edges are assigned to these planes so that no edges overlap or cross. The vertices are stretched into segments of $z$-lines.

\subsection{Drawings of $O\left(n^{3}\right)$ volume for $k=1$}

In this section, we describe two strategies to draw $K_{n}$ with at most $k=1$ bends on any edge. The first layout scheme draws $K_{n}$ in an $n \times n \times n$-grid. The second scheme then makes two drawings of $K_{n / 2}$ (without recursion) using the first scheme; then it positions these drawings in an $\frac{n}{2} \times n \times \frac{n}{2}$-grid and supplies the edges between the two parts. For simplicity, assume below that $n$ is divisible by 4 .

Drawing $K_{\boldsymbol{n}}$ in an $\boldsymbol{n} \times \boldsymbol{n} \times \boldsymbol{n}$-grid for $\boldsymbol{k}=\mathbf{1}$ Enumerate the vertices as $v_{1}, \ldots, v_{n}$. Place vertex $v_{i}$ at $(i, i)$. Route edge $e=\left(v_{i}, v_{j}\right)$, where $i<j$, with one bend via $(i, i),(i, j),(j, j)$. Note that no vertex or part of an edge is placed at a point $(x, y)$ with $y<x$.

Now partition the edges of $K_{n}$ into edge sets $E_{i}^{a}, E_{i}^{b}, i=1, \ldots, \frac{n}{2}$, defined as $E_{i}^{a}=\left\{\left(v_{i-l+1}, v_{i+l}\right) \mid l=1, \ldots, \frac{n}{2}\right\}$ and $E_{i}^{b}=\left\{\left(v_{i-l}, v_{i+l}\right) \mid l=1, \ldots, \frac{n}{2}-1\right\}$ (all additions are modulo $n$ ). It is easy to check that these sets indeed partition the edges of $K_{n}$, and that no crossings or overlaps occur among edges in $E_{i}^{a}$ nor among edges in $E_{i}^{b}$. Hence only $n z$-planes are needed. See Fig. 2 . This gives the following lemma.

Lemma 3. There exists a drawing of $K_{n}$ in an $n \times n \times n$-grid with one bend per edge such that the points $\{(x, y, z): y<x\}$ are unused. 

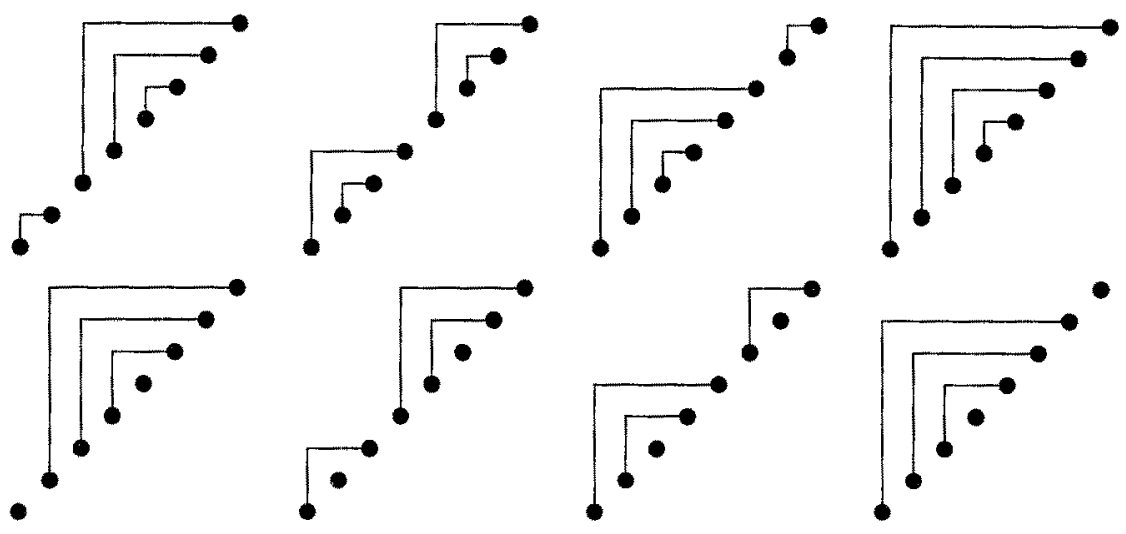

Fig. 2. The sets $E_{b}^{1}, \ldots, E_{b}^{4}$ for $K_{8}$.

Remark: Note that $E_{i}^{a}$ and $E_{i}^{b}$ can be drawn in the same plane by reflecting the edges of $E_{i}^{a}$ with respect to the diagonal line through the vertices. This yields a drawing of $K_{n}$ in an $n \times n \times \frac{n}{2}$-grid. This strategy is closely related to the pagenumber of a graph and in fact, may prove a useful idea for drawing sparse graphs. This idea yields, for example, a method for drawing planar graphs in $O\left(n^{2}\right)$ volume in an $n \times n \times 4$-grid, since it is known that planar graphs have pagenumber equal to 4 (see [Yan89]).

Drawing $K_{n}$ in an $\frac{n}{2} \times n \times \frac{n}{2}$-grid for $k=1$ Let $K^{1}$ and $K^{2}$ denote two drawings of $K_{n / 2}$ as described in the previous lemma. Thus each drawing has an $\frac{n}{2} \times \frac{n}{2} \times \frac{n}{2}$ bounding box. Reflect the points in the box for $K^{2}$ through the $(y=0)$-plane, so that all points in the reflected $K^{2}$ have negative $y$-coordinate. Then rotate this reflected $K^{2}$ so that vertex $v_{j}$ of the rotated, reflected $K^{2}$ overlaps the points $(x,-j, j)$, where $1 \leq x \leq \frac{n}{2}$. See Fig. 3 .

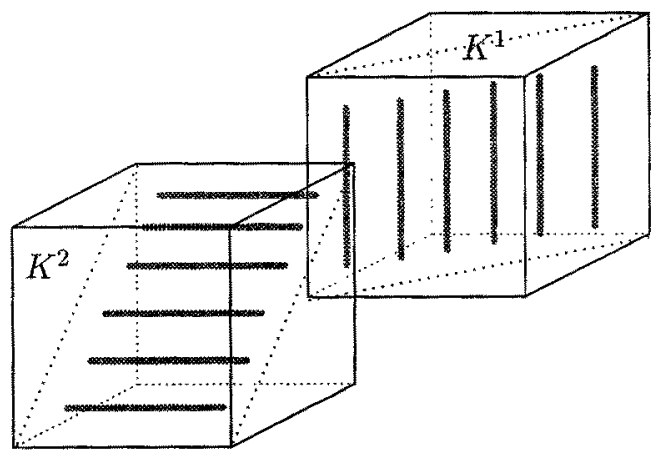

Fig. 3. $K_{1}$ and $K_{2}$ 
Each vertex $v_{i}$ in $K^{1}$ sees each vertex $v_{j}$ in the rotated, reflected $K^{2}$ along the $y$-line segment $[(i, i, j),(i,-j, j)]$. Therefore, these edges can be drawn as straight line segments, thus producing a drawing of $K_{n}$. Delete the unused $y$ plane of $y$-coordinate 0 to obtain a drawing with dimensions $X=Z=\frac{n}{2}$ and $Y=n$. There are $n^{2} / 4$ edges drawn without a bend, and all other edges have one bend, so the total number of bends is $n^{2} / 4-n / 2$.

Theorem 4. $K_{n}$ can be drawn in a $\frac{n}{2} \times n \times \frac{n}{2}$-grid with at most one bend per edge and a total number of bends equal to $n^{2} / 4-n / 2$.

\subsection{A smaller $O\left(n^{3}\right)$ volume drawing for $k=2$}

A similar strategy can be applied when a maximum of $k=2$ bends on an edge is allowed. In this section, $K_{n}$ is drawn with at most two bends per edge by first making two copies of a drawing for $K_{\frac{n}{2}}$ and then placing them in a grid of side-length $\frac{n}{2}$ and supplying the edges connecting the two parts.

Drawing in an $n \times \frac{n}{2} \times n$-grid Enumerate the vertices as $\left\{v_{1}, \ldots, v_{n}\right\}$ and place $v_{i}$ at $(x, y)=(i, 1)$ in a 2-D $(x, y)$-plane. To route edge $e=\left(v_{i}, v_{j}\right)$, where $i<j$, let $y=\left\lceil\frac{j-i}{2}\right\rceil$ and route $e$ via the points $(i, 1),(i, y),(j, y),(j, 1)$, creating two bends if $y>1$ and no bends if $y=1$.

Define the edge sets $E_{i}^{a}$ and $E_{i}^{b}$ as above. Again there are no crossings nor overlaps among edges in the same set and so $n z$-planes suffice. Since the largest $y$-coordinate is $\left\lceil\frac{n-1}{2}\right\rceil$, the bounding box has dimensions $n \times \frac{n}{2} \times n$. The edges $\left(v_{i}, v_{i+1}\right)$ for $i=1, \ldots, n-1$ are drawn straight; all other edges have two bends, so the total number of bends is $n^{2}-3 n+2$.

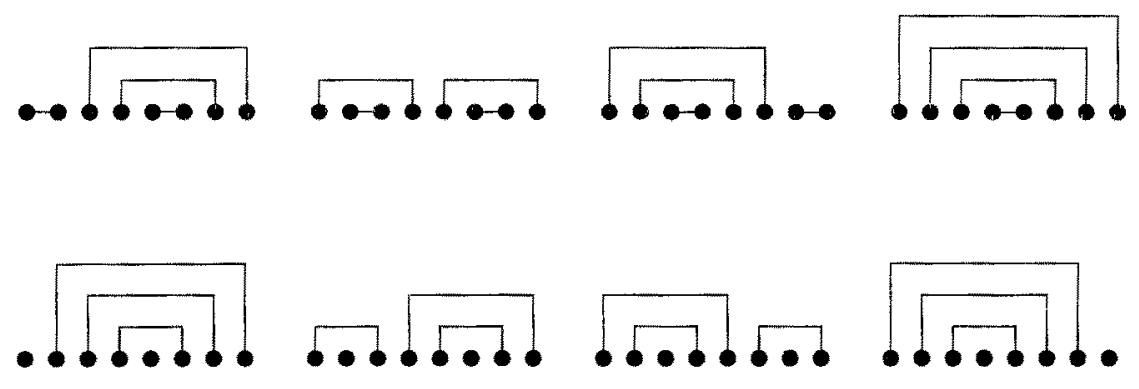

Fig. 4. The edge sets of $K_{8}$ drawn with at most two bends per edge.

Lemma 5. The graph $K_{n}$ can be drawn in an $n \times \frac{n}{2} \times n$-grid, with a total of $n^{2}-3 n+2$ bends and at most two bends per edge, such that vertex $v_{i}$ overlaps the points $(i, 1, z)$, where $1 \leq z \leq n$. 
Drawing in an $\frac{n}{2} \times \frac{n}{2} \times \frac{n}{2}$-grid Let $K^{1}$ and $K^{2}$ denote two $K_{\frac{n}{2}}$ 's drawn as described above. Thus each drawing has a bounding box of dimensions $\frac{n}{2} \times \frac{n}{4} \times \frac{n}{2}$. Reflect $K^{2}$ through the $(y=0)$-plane, so that all points in the reflected $K^{2}$ have negative $y$-coordinate. Then rotate the reflected $K^{2}$ so that vertex $v_{j}$ of the rotated, reflected $K^{2}$ now overlaps the points $(x,-1, j)$, where $1 \leq x \leq \frac{n}{2}$.

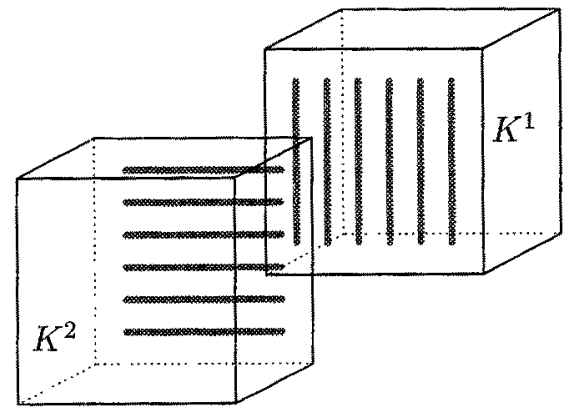

Fig. 5. Two $K_{\frac{n}{2}}$ 's, with $K^{2}$ reflected and rotated

Each vertex $v_{i}$ in $K^{1}$ sees each vertex $v_{j}$ in the rotated, reflected $K^{2}$ along the $y$-line segment $[(i, 1, j),(i,-1, j)]$. Therefore, these edges can be drawn as straight lines, thus producing a drawing of $K_{n}$. Removing the unused $y$-plane of $y$-coordinate 0 yields a drawing of dimensions $X=Y=Z=\frac{n}{2}$. The total number of bends is $2\left(n^{2} / 4-\frac{3}{2} n+2\right)=n^{2} / 2-3 n+4$.

Theorem 6. $K_{n}$ can be drawn in an $\frac{n}{2} \times \frac{n}{2} \times \frac{n}{2}$-grid with a total of $n^{2} / 2-3 n+4$ bends and at most two bends per edge.

\subsection{An $O\left(n^{2.5}\right)$ Volume drawing for $k=3$}

In this section, we draw $K_{n}$ with at most $k=3$ bends on any edge and with volume $\mathcal{O}\left(n^{2.5}\right)$. Case 2 of the lower bound proof suggests what general form such a drawing might take. For simplicity, assume below that $n=N^{2}$ for some integer $N$. Enumerate the vertices as ordered pairs $(i, j)$, where $1 \leq i \leq N$, $1 \leq j \leq N$, and place vertex $(i, j)$ at $(2 i, 2 j)$ in the 2-D $x, y$-plane. Suppose edge $e$ joins vertex $\left(i_{1}, j_{1}\right)$ and vertex $\left(i_{2}, j_{2}\right)$. After possible renaming, we may assume that $i_{1} \leq i_{2}$, and that if $i_{1}=i_{2}$, then $j_{1}>j_{2}$. Call $e$ an $L$-edge if $j_{1}>j_{2}$ and a $\Gamma$-edge otherwise.

Initially route each $L$-edge via the points $\left(2 i_{1}, 2 j_{1}\right),\left(2 i_{1}+1,2 j_{1}\right),\left(2 i_{1}+1,2 j_{2}+\right.$ 1), $\left(2 i_{2}, 2 j_{2}+1\right),\left(2 i_{2}, 2 j_{2}\right)$, thus with three bends. Route each $\Gamma$-edge via points $\left(2 i_{1}, 2 j_{1}\right),\left(2 i_{1}+1,2 j_{1}\right),\left(2 i_{1}+1,2 j_{2}-1\right),\left(2 i_{2}, 2 j_{2}-1\right),\left(2 i_{2}, 2 j_{2}\right)$.

Split the L-edges into $N(N-1)$ groups $E_{d_{x}, d_{y}}$, with $0 \leq d_{x} \leq N-1$ and $1 \leq d_{y} \leq N-1$. Each group $E_{d_{x}, d_{y}}$ consists of those edges $\left(\left(i_{1}, j_{1}\right),\left(i_{2}, j_{2}\right)\right)$ for which $i_{2}=i_{1}+d_{x}$ and $j_{2}=j_{1}-d_{y}$. These groups cover all L-edges since $i_{1} \leq i_{2}$ and $j_{1}>j_{2}$ for any L-edge. 
Now split each group $E_{d_{x}, d_{y}}$ into at most $d_{x}+d_{y}$ sets of edges as follows. For $p=0, \ldots, d_{x}+d_{y}-1$, let $E_{d_{x}, d_{y}}^{p}$ be the edges in $E_{d_{x}, d_{y}}$ for which $j_{2}-i_{1}=p$ modulo $\left(d_{x}+d_{y}\right)$. In other words, the lower left "corners" of the L-edges in $E_{d_{x}, d_{y}}^{p}$ lie on diagonals that intersect the $y$-axis at the value $2 p$ modulo $\left(2 d_{x}+2 d_{y}\right)$. See Fig. 6. It is easy to check that no two edges in $E_{d_{x}, d_{y}}^{p}$ overlap or intersect since the corners of the L's are placed on a sequence of diagonals having a vertical spacing of $2\left(d_{x}+d_{y}\right)$ between adjacent diagonals. Also, note that $E_{d_{x}, d_{y}}^{p}$ is non-empty only if $p \leq 2 N-d_{x}-d_{y}{ }^{5}$
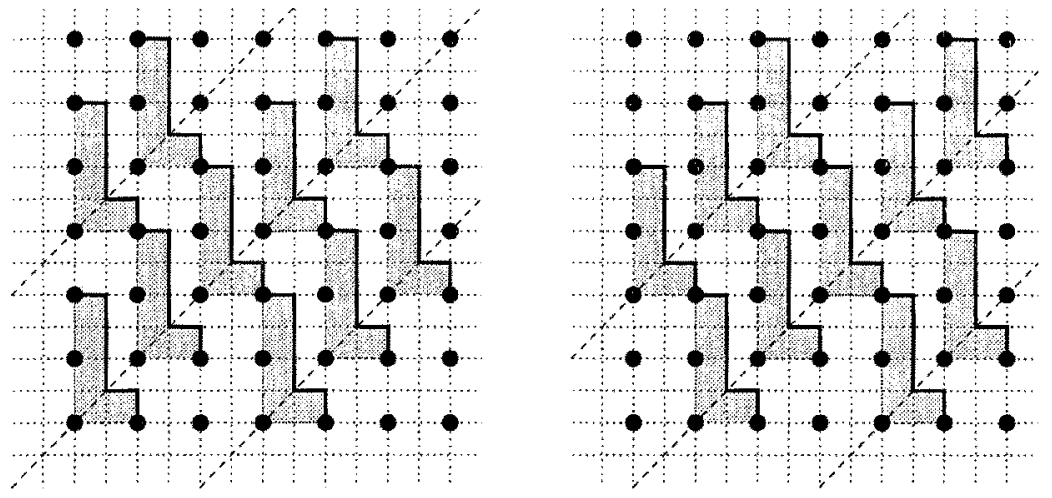

Fig. 6. The edge sets $E_{1,2}^{0}$ and $E_{1,2}^{2}$.

Assign a $z$-plane to each set $E_{d_{x}, d_{y}}^{p}$ to obtain a legal drawing of the L-edges. Route the $\Gamma$ edges in an analogous fashion. This doubles the number of $z$-planes, yielding a drawing of $K_{n}$ in a grid with $X=Y=2 N=2 \sqrt{n}$. The $Z$ dimension is given by

$$
2 \sum_{d_{x}=0}^{N-1} \sum_{d_{y}=1}^{N-1} \min \left\{d_{x}+d_{y}, 2 N-d_{x}-d_{y}\right\}
$$

Some analysis shows that this sum is at most

$2\left[\sum_{k=1}^{N-1} k(2 k-1)+(N-1) N\right]=\frac{2(N-1) N(2 N-1)}{3}-(N-1) N+2(N-1) N$

which is less than $\frac{4}{3} N^{3}$. Every edge has three bends. However, the $2 N(N-1)=$ $2 n-2 \sqrt{n}$ edges where $d_{x}=0$ and $d_{y}=1$, or $d_{x}=1$ and $d_{y}=0$ can be drawn without a bend. So the total number of bends is $3\left(n^{2} / 2-n / 2\right)-3(2 n-2 \sqrt{n})=$ $\frac{3}{2} n^{2}-\frac{15}{2} n+6 \sqrt{n}$.

\footnotetext{
${ }^{5}$ A java applet demonstrating the sets and their routings for $K_{100}$ can be found at http://ww.cs.uleth.ca/ wismath/ortho.html.
} 
Theorem 7. If $n=N^{2}$ is a square, then $K_{n}$ can be drawn in a $2 N \times 2 N \times \frac{4}{3} N^{3}$ grid (so volume $\frac{16}{3} n^{2.5}$ ) with $\frac{3}{2} n^{2}-\frac{15}{2} n+6 \sqrt{n}$ bends and at most three bends per edge.

\section{Conclusions}

This paper is one of the first to address volume and bend considerations for 3-D orthogonal grid drawings of graphs. The focus has been on $K_{n}$, since it is the most difficult graph on $n$ vertices to draw in small volume or with restrictions on bends. In particular, we have

- provided a method for drawing $K_{n}$ with volume that is provably within a constant factor (same constant for all $n$ ) of best possible in the case that at most $k$ bends per edge are allowed, where $k \geq 3$;

- proved the non-existence of drawings of $K_{n}$ for large $n$ in the $k=0$ case, where no bends are permitted;

- proved a lower bound of $\Omega\left(n^{2.5}\right)$ and an upper bound of $O\left(n^{3}\right)$ on the volume of drawings of $K_{n}$ when $k=1$ and $k=2$.

An open problem is to close the gap between the upper and lower bounds in the $k=1$ and $k=2$ cases, where at most 1 and at most 2 bends on each edge are permitted, respectively. The ideas and methods presented here may serve as a useful starting point for constructing drawings with good constant factors for volume and bends.

\section{Acknowledgments}

Thanks to Michael Kaufmann for discussions on orthogonal drawings. The joint results of this paper have also appeared as part of the PhD thesis of T. Biedl at Rutgers University. 


\section{References}

[BEFLMRSW93] P. Bose, H. Everett, S. Fekete, A. Lubiw, H. Meijer, K. Romanik, T. Shermer and $\mathrm{S}$. Whitesides. On a visibility representation for graphs in three dimensions, Proc. Graph Drawing '93, Paris, 1993, 38-39.

[BMT97] T. Biedl, B. Madden, and I. Tollis. The three-phase method: A unified approach to orthogonal graph drawing. In these proceedings.

[Bra96] F. Brandenburg, ed. Symposium on Graph Drawing '95, Passau, Germany, Springer-Verlag LNCS 1027, 1996.

[ESW97] P. Eades, A. Symvonis and S. Whitesides. Two algorithms for three dimensional orthogonal graph drawing. In S. North, ed. Symposium on Graph Drawing '96, Berkeley, California, Springer Verlag LNCS 1190, 1997, 139-154.

[FHW96] S. Fekete, M. Houle and S. Whitesides. New results on a visibility representation of graphs in 3d. In [Bra96], 234-241.

[FK96] U. Fößmeier and M. Kaufmann. Drawing high degree graphs with low bend numbers. In [Bra96], 254-266.

[GRS80] R. Graham, B.L. Rothschild and J. Spencer. Ramsey Theory. John Wiley, 1980.

[PT96] A. Papakostas and I. Tollis. High-degree orthogonal drawings with small gridsize and few bends. In 5th Workshop on Algorithms and Data Structures, Springer Verlag LNCS, 1997. To appear.

[PT97] A. Papakostas and I. Tollis. Incremental orthogonal graph drawing in three dimensions. In these proceedings.

[Ros83] A. Rosenberg. Three-dimensional VLSI: A case study. Journal of the Association of Computing Machinery, 30 (3), 1983, 397-416.

[TT86] R. Tamassia and I. Tollis. A unified approach to visibility representations of planar graphs. J. of Discrete and Computational Geometry 1, 1986, 321-341.

[Wis85] S. K. Wismath. Characterizing bar line-of-sight graphs. Proc. of the 1st ACM Symp. on Computational Geometry, Baltimore, Maryland, USA, 1985, 147-152.

[Yan89] M. Yannakakis, Embedding planar graphs in four pages. 18th Annual ACM Symposium on Theory of Computing, J. Comput. System Sci. 38 (1989), no. 1, $36-67$. 